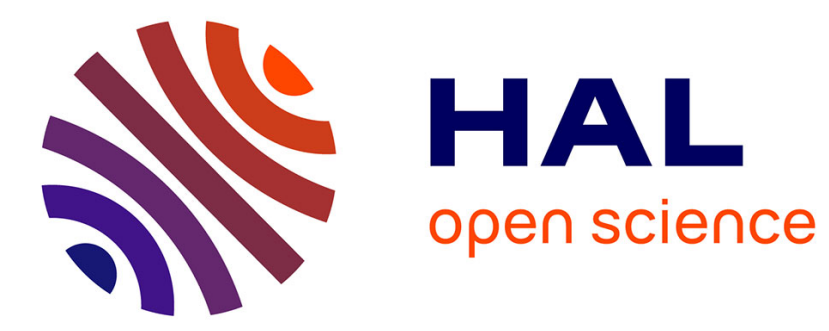

\title{
A propos de deux opérations de dénombrement
}

Jean Brossier

\section{To cite this version:}

Jean Brossier. A propos de deux opérations de dénombrement. Revue forestière française, 1962, 10, pp.854-859. 10.4267/2042/24417 . hal-03386344

\section{HAL Id: hal-03386344 \\ https://hal.science/hal-03386344}

Submitted on 19 Oct 2021

HAL is a multi-disciplinary open access archive for the deposit and dissemination of scientific research documents, whether they are published or not. The documents may come from teaching and research institutions in France or abroad, or from public or private research centers.
L'archive ouverte pluridisciplinaire HAL, est destinée au dépôt et à la diffusion de documents scientifiques de niveau recherche, publiés ou non, émanant des établissements d'enseignement et de recherche français ou étrangers, des laboratoires publics ou privés. 


\title{
A PROPOS DE DEUX OPÉRATIONS DE DENOMBREMENT
}

\author{
PAR \\ J. BROSSIER \\ Ingénieur des Eaux et Forêts à Grenoble
}

M'intéressant depuis quinze années au problème de la protection du chamois dans le massif de la Grande Chartreuse, j'avais souvent pensé au dénombrement du cheptel qui permettrait de suivre l'efficacité du gardiennage, la vitalité de l'espèce, les migrations. etc... Dans un secteur aussi boisé, cette numération n'est pas impossible, mais elle est très délicate et suppose un nombre important d'excellents observateurs. Aussi n'est-il pas imaginable de réunir les moyens nécessaires.

Un bref séjour dans le Briançonnais, secteur le plus riche sans doute en chamois de toutes les Alpes françaises, m'a permis de réaliser cette opération de dénombrement dans deux réserves. Qu'il s'agisse du parc national du Pelvoux dans sa partie Vallouise, surveillé par l'Administration forestière ou du massif du Combeynot dépendant de la Fédération de chasse des Hautes-Alpes - nous avions à étudier des territoires de forte altitude (1 500 à 4000 mètres pour l'un, 1600 à 3000 mètres pour l'autre), ne présentant qu'une faible surface couverte de forêts. L'observation des hardes à la jumelle est commode dans ces terrains souvent nus, longtemps enneigés; en profitant de l'époque éminemment favorable du rassemblement des hardes pour le rut (d'octobre à décembre), il semblait possible d'opérer avec précision; je disposais surtout dans 1'Inspection de Briançon d'une équipe exceptionnelle de préposés montagnards, chasseurs de chamois pour certains, ce qui constituait l'atout majeur pour réussir.

Les critiques faites au parc du Pelvoux, justifiées pour une part, m'avaient semblé très systématiques dans certains milieux; en 1961, prenant contact avec la Vallouise, j'avais découvert dans le vallon de Celse Nière non seulement un territoire aux possibilités excep- 


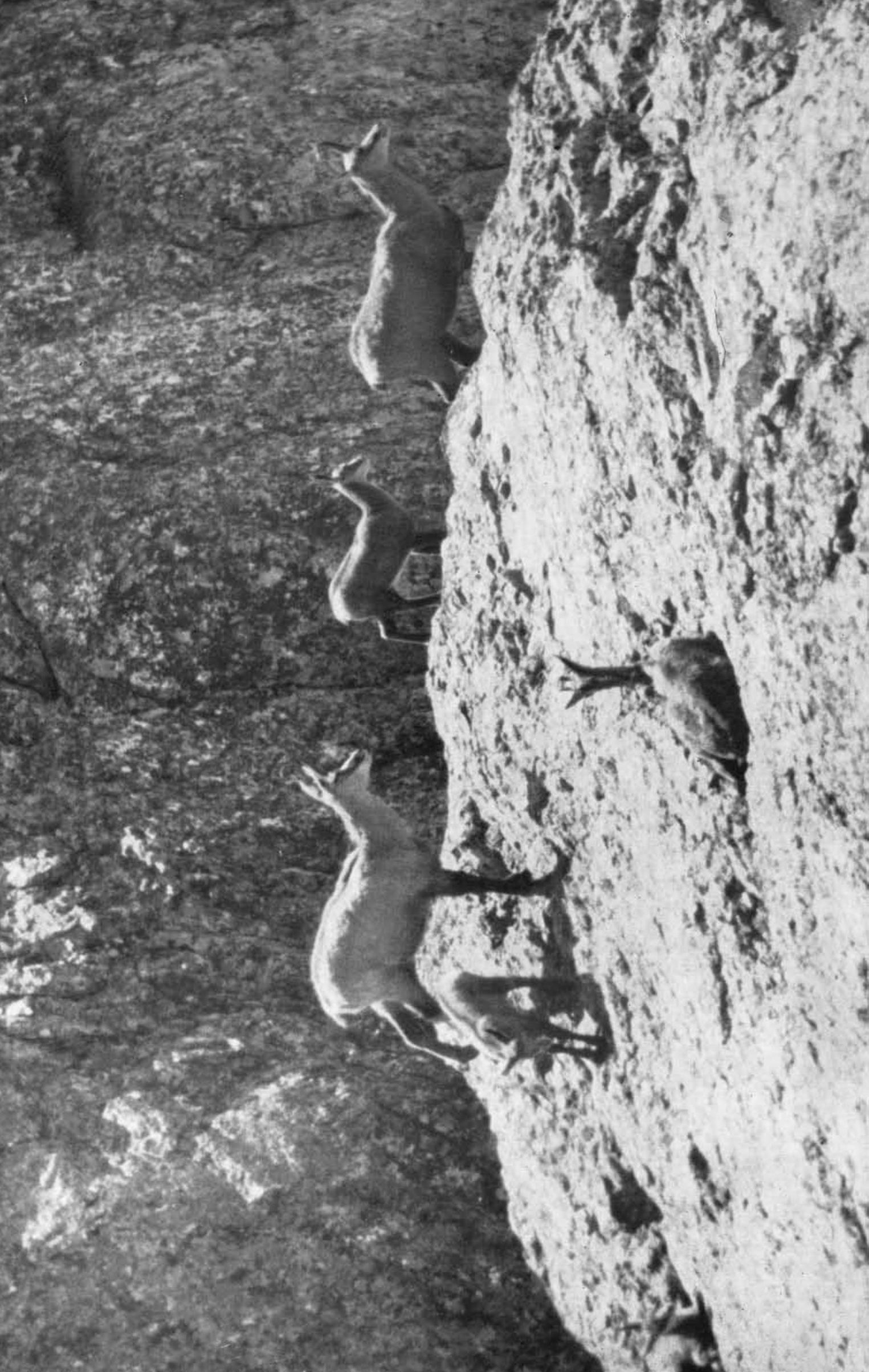




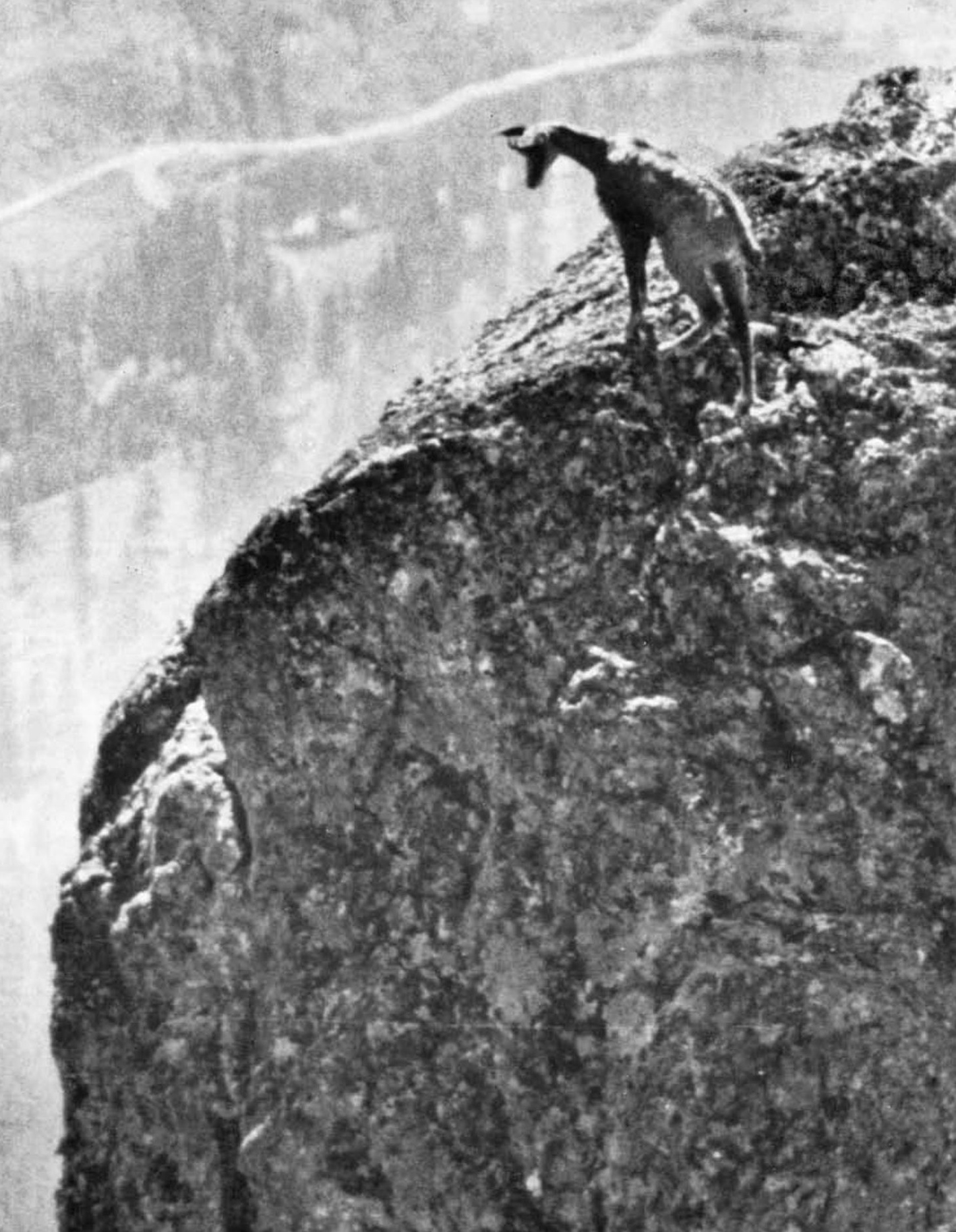


tionnelles, biotope idéal du chamois, mais aussi une population effective qui m'avait paru, après quelques sorties, dépasser sensiblement les estimations prudemment avancées. Sur ces entrefaites, le jour de la retraite sonnait pour Pierre EngILBERge, braconnier repenti et père du parc: son fils. Maurice lui succédait dans l'été 1961 et je pensais alors à faire un véritable inventaire qui serait pour Engilberge, “ l'ancien », un bilan de fin d'exercice. C'est ainsi que fut décidé notre premier dénombrement.

Il eut lieu le 26 octobre. Le temps, un peu perturbé la veille, voulut bien nous favoriser; nous fûmes gratifiés d'une de ces journées exceptionnelles de l'automne briançonnais. Quatre équipes seulement se divisèrent le territoire peuplé. Sur les 5000 hectares du parc situés en Vallouise, moins de 1500 hectares sont habités à

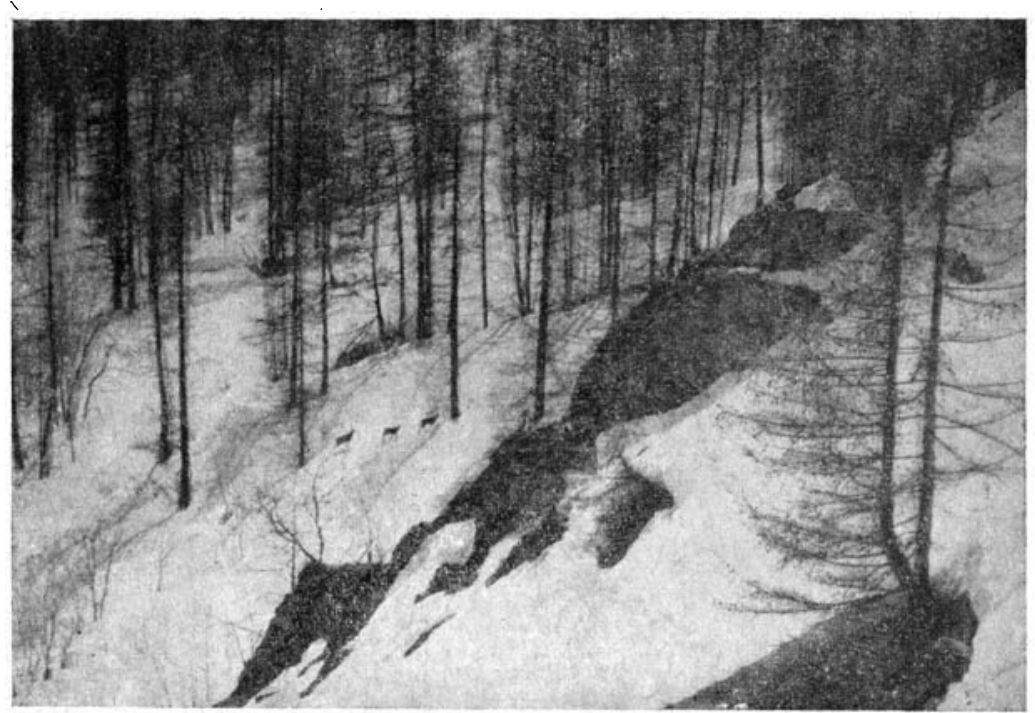

l'automne: une chute de neige récente semblait avoir contraint les hardes à descendre au-dessous de 2700 mètres. Leur localisation avait été reconnue dans la semaine précédente. Restait, pour chaque équipe de deux observateurs, à parcourir le secteur assigné en " découvrant ) à distance favorable pour compter soigneusement sans déranger les chamois. La configuration du terrain permettait, en outre, à certaines équipes de contrôler de plus loin le travail de leurs voisins.

La chance fut avec nous. Malgré une dissémination des animaux importante, nous prétendons avoir recensé la presque totalité des 
chamois de chaque secteur. Aucune confusion ne fut possible; un chamois se déplaçant n'aurait pu être compté par deux observateurs différents, l'heure de chaque mouvement étant soigneusement noté: Un léger doute demeure sur le nombre trouvé dans les faces Sud des Ecrins à la Grande Sagne: l'angle de vue est assez difficile à partir des rives du Glacier Noir et il faudrait prévoir désormais une équipe pour ce seul secteur.

Nous pûmes ainsi garantir que le parc contenait 207 chamois: premier résultat satisfaisant au regard des railleries qui s'expliquaient en 1945 au sortir des années de terrible braconnage, mais

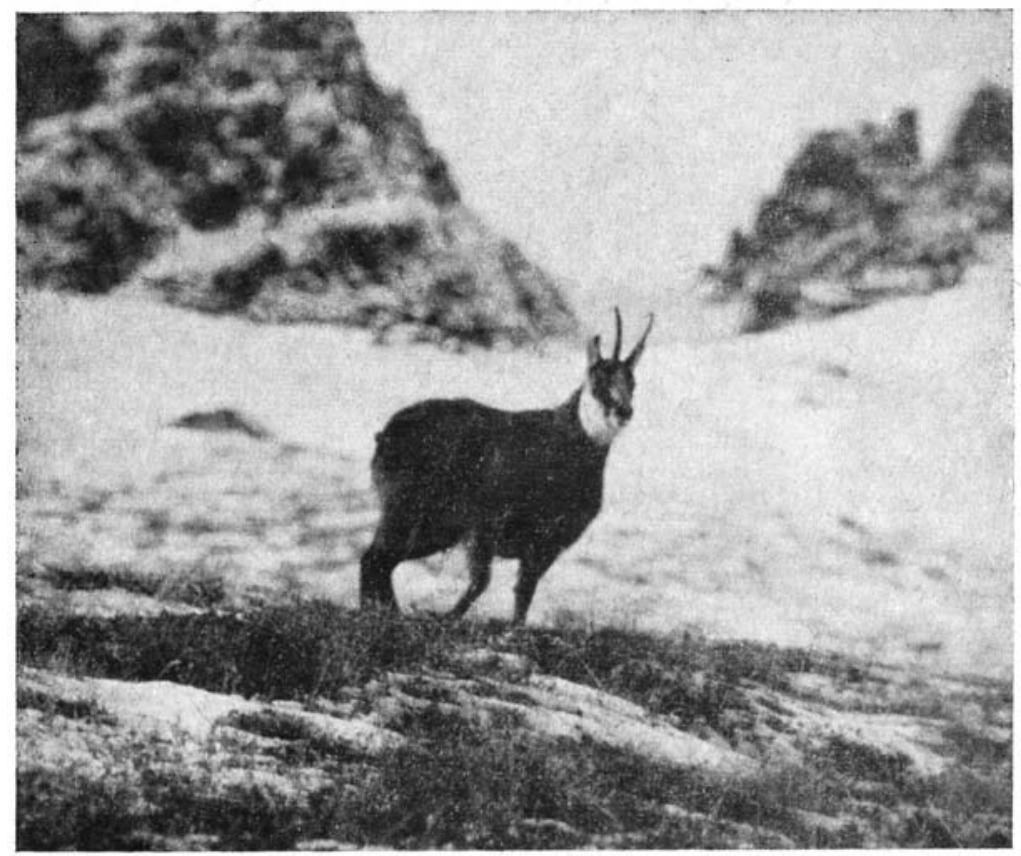

n'ont désormais plus de sens. Espoir très grand, car, sauf catastrophe, ces 200 chamois doivent devenir 400 en moins de cinq ans, si les possibilités d'alimentation du secteur sont assez grandes et si l'on réalise l'augmentation de surface prévue sur la Commune de Vallouise notamment.

Après cet essai, il parut intéressant de clénombrer le célèbre Conbeynot, créé par les efforts des locataires successifs, notamment de $M$. de Gournay, efforts appuyés et relayés par la Fédération de chasse des Hautes-Alpes sous l'impulsion de son président, M. REYDELLET. 
Cette opération fut décidée en plein accord avec ce dernier: forestiers et fédéraux y collaborèrent, chose rare, mais combien souhaitable!

L'étendue du territoire (près de 5000 hectares), la brièveté des jours et la difficulté du terrain nous amenèrent à prévoir 7 équipes de deux observateurs. Ce nombre s'avéra suffisant. Grâce à la parfaite connaissance du terrain de l'Agent Technique Peyron, du Monétier et des gardes du Combeynot, l'opération se déroula parfaitement. Une petite harde en escapade sur l'arête supérieure ne put être exactement contrôlée. Le nombre de 405 chamois identifiés est donc légèrement inférieur à la réalité.

On peut être assuré, dès maintenant, que cette réserve a « fait le plein ». L'excédent des naissances ne se traduit plus par une augmentation du troupeau à l'intérieur du massif, mais par une émigration dans les territoires environnants sur Villar-d'Arène, Monétier-les-Bains, voire Pelvoux.

Quelle conclusion tirer de ces expériences qui n'ont rien d'inédit? Leur résultat m'a surtout confirmé que l'on sous-estime toujours la population d'un massif et que l'on peut désormais être résolument optimiste sur l'avenir du chamois.

Dans la majeure partie des Alpes françaises - hormis quelques massifs trop courus - le chamois est en pleine progression. Les braconniers sont moins actifs: les plus terribles ont vieilli ou sont morts sans avoir été remplacés. Les montagnards ont des activités plus régulières qui ne leur laissent plus ces longues périodes d'inaction qu'il fallait bien meubler. La majeure partie des chasseurs n'a plus la résistance et l'opinâtreté dans l'effort qui font le chasseur de chamois. Les jeunes, dans les chasses en groupe, ne laissent-ils pas aux vieux le soin de la traque, préférant " prendre le poste ", travail moins fatigant, mais combien moins passionnant?

Certes, le chamois a devant lui un brillant avenir et le développement des routes n'est pas un danger trop grave pour lui. Les amis de la montagne peuvent se réjouir; ils admirent déjà facilement nos indigènes antilopes au cours de nombres d'ascensions du Taillefer au Pelvoux, des " soms » de Chartreuse ou des Bauges aux pics du Haut Queyras.

Le législateur ne doit pas, pour autant, desserrer sa pression. Une densité supérieure est souhaitable; certains massifs (Vercors, Belledonne, etc...) restent pauvres.

Les règlements devraient cependant être assouplis. Les restrictions ne devraient porter que sur la durée de l'ouverture limitée à 3 ou 4 semaines. Le maintien de belles réserves est la mesure la plus souhaitable. 
Pour résoudre l'épineux problème de la réglementation des armes, on gagnerait à se montrer assez indulgent; l'usage de la chevrotine, cause de trop de blessures mortelles n'arrêtant pas le chamois, devrait pourtant être aboli; le tir de la balle dans les canons lisses du fusil de chasse laisserait plus de chances à l'animal.

Que dire de l'interdiction du chevreau, souhaitable dans son principe, mais combien discuté? Que dire encore de l'interdiction de la " chèvre suitée ": animal dépourvu de toute carte d'identité dont la particularité est précisément de perdre son caractère, une fois abattu. Quel tribunal sera assez sévère et compétent en connaissances biologiques pour admettre qu'une femelle abattue en lactation

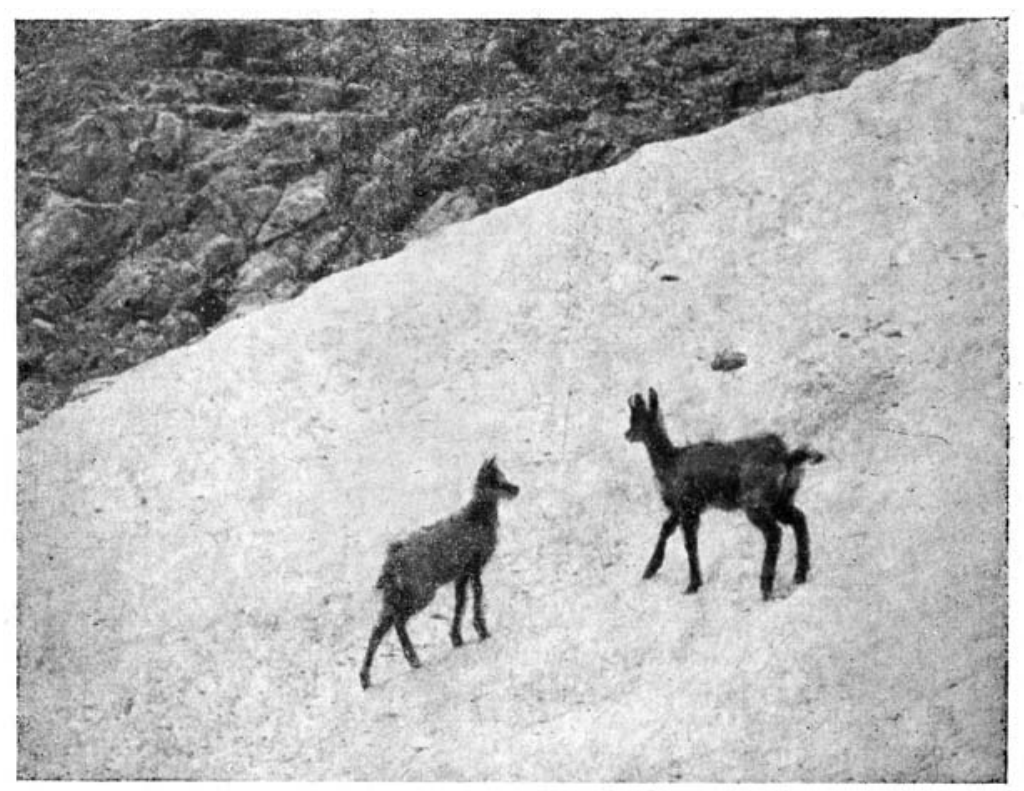

ne peut s'être présentée au chasseur que flanquée de son chevreau? Dans un département où existe une telle interdiction, on mettrait la révolution si, au soir de l'ouverture, forestiers et gendarmes verbalisaient les qui de droit ayant commis une infraction à ce point de l'Arrêté!

Le législateur, abusé par trop de conseillers, souvent braconniers de surcroit, n'a pas pesé, bien souvent, les possibilités d'application de règlements théoriquement parfaits. Revenons au bon sens! Nous verserons un pleur sur le chevreau lâchement abattu par un chasseur sans pudeur sur le cadavre de sa mère; le vrai chasseur n'a 
pas besoin, lui, d'Arrêté pour résister à la tentation du massacre. Nous regretterons que la traque permette à quelque citadin bedonnant d'abattre un animal dont la conquête devrait être réservée aux (" purs ). Mais pourquoi interdire un tel mode de chasse, seul praticable dans nombre de secteurs forestiers de nos Alpes?

Peut-être verrons-nous peu à peu les chasseurs d'image remplacer les " tueurs ». A l'exemple des Bille, Vaucher et Samiver, ils goûteront la joie profonde que nos dégustions quand, dans nos jumelles, nous prétendions compter, tels des moutons sur les hautsalpages, les chamois du Parc et ceux du Combeynot.

Les clichés sont de « Georges Rollet, Photopress, Grenoble ». 